\title{
AN IDENTITY PROPERTY FOR 2-COMPLEX PAIRS
}

\author{
MICHAEL P. HITCHMAN \\ Department of Mathematical Sciences, Lewis \& Clark College, Portland, OR 97219, USA
}

(Received 17 August, 1997)

\begin{abstract}
An identity property defined for a pair of 2-complexes $(Y, X)$ first arose in 1993 within a strategy for constructing a counterexample of infinite type to Whitehead's Asphericity Conjecture. In this note we make use of the theory of pictures to characterize a more general right $N$-identity property, where $N<\pi_{1} Y$. We also define combinatorial asphericity (CA) for the pair $(Y, X)$ and determine a test for (CA) in the case that $Y$ is obtained from $X$ by the addition of a single 2-cell. This test can be used to determine an explicit generating set for $\pi_{2} Y$.
\end{abstract}

1991 Mathematics Subject Classification. 57M20.

1. Introduction. In this note we study an identity property for a pair of 2complexes, $(Y, X)$, where $Y$ is obtained from $X$ by the addition of 2-cells. This property is a natural generalization of the (absolute) identity property for a 2complex and first arose in the context of a question of J.H.C. Whitehead [15]: is every subcomplex of a connected, aspherical 2-complex itself aspherical? Much research has been conducted regarding this still unanswered question (see [4] for a good survey of this research). One strategy for building a counterexample to the Whitehead conjecture (which asserts the answer to his question is "yes") is to construct an infinite chain of 2-complexes $X_{1} \subset X_{2} \subset \cdots X_{i} \subset X_{i+1} \subset \cdots$ in which $\pi_{2} X_{1}$ is not trivial, but $\pi_{2}\left(X_{i}\right) \rightarrow \pi_{2}\left(X_{i+1}\right)$ is trivial for each $i \geq 1$ (see [12]). In [8], Dyer introduces the identity property within a strategy for constructing such a chain of spaces. In this strategy, the identity property is used to replace the homotopy requirement $\left(\pi_{2} X_{i} \stackrel{0}{\rightarrow} \pi_{2} X_{i+1}\right)$ with a homological one that is perhaps more accessible. For the reader's convenience we describe this strategy in some detail below, in Section 3.

We may recover the (absolute) identity property for a 2-complex $Y$ by considering the pair $\left(Y, Y^{(1)}\right)$, where $Y^{(1)}$ is the 1-skeleton of $Y$. This absolute identity property is the traditional way to detect asphericity of a 2-complex. More generally, if $N$ is a subgroup of the fundamental group of $Y$, the right $N$-identity property approximates asphericity. That is, $Y$ has the right $N$-identity property if and only if $\pi_{2} Y \rightarrow H_{2} Y_{N}$ is trivial, where $Y_{N}$ is the cover of $Y$ corresponding to $N$. The right $N$ identity property has been the focus of much attention (see [7] for a good survey article), and it arises naturally in the context of the Whitehead conjecture. A deep result of J.F. Adams [1] says that a subcomplex $X$ of an aspherical 2-complex has the right $P$-identity property, for some perfect subgroup $P$ of the fundamental group of $X$.

In this paper we use pictures to define a right $N$-identity property for the pair $(Y, X)$, where $N$ is a subgroup of $\pi_{1} Y$. We will always view our 2-complexes as arising from group presentations in the standard way, and it is often convenient to discuss the identity property in terms of a pair of group presentations $(\mathcal{Q}, \mathcal{P})$ where $\mathcal{Q}$ is obtained from $\mathcal{P}$ by the addition of relators. In Section 3 we present various characterizations of the $\mathrm{N}$-identity property that extend known characterizations of 
the absolute identity property. In Section 4 we make use of a Cockcroft property on certain disk pictures to determine a combinatorial group-theoretic characterization of the identity property.

In the last two sections we generalize to 2-complex pairs $(Y, X)$ the notion of weakening the asphericity of a 2-complex to combinatorial asphericity. Simple mixed pictures called relative dipoles play an important role here. We define $(Y, X)$ to be combinatorially aspherical (CA) if $\pi_{2} Y$ is generated (over $\pi_{2} X$ ) by a set of relative dipoles. In the case $Y$ is obtained from $X$ by adding a single 2-cell, we prove a simple test for determining an explicit set of generators for $\pi_{2} Y$ (over $\pi_{2} X$ ).

2. Pictures. If $Y$ is the model of the presentation $\mathcal{Q}=\langle\mathbf{x}: \mathbf{r}, \mathbf{s}\rangle$, then elements of $\pi_{2} Y$ are represented by spherical pictures over $\mathcal{Q}$. We refer the reader to [5] and [13] for two thorough treatments of pictures, but we outline some key features here.

Any oriented transverse path $\gamma$ in a picture $\mathbf{B}$ defined over $\mathcal{Q}$ determines a word, $\omega(\gamma) \in F(\mathbf{x})$, from the labels of the arcs it traverses. If $\gamma_{i}$ and $\gamma_{i}^{\prime}$ are distinct transverse paths from the global basepoint of $\mathbf{B}$ to the basepoint of a disk $\Delta_{i}$ in $\mathbf{B}$, then they may determine distinct words in $F$. However, an important feature of pictures is that these words determine the same element of the group presented. This fact is essentially due to a pictorial version of van Kampen's Lemma.

Lemma 2.1. (van Kampen) Suppose $\mathcal{P}=\langle\mathbf{x}: \mathbf{r}\rangle$ presents the group G. For any word $w \in F(\mathbf{x})$, there exists a picture $\mathbf{B}$ over $\mathcal{P}$ with $\partial \mathbf{B}=w$ if and only if $w$ has trivial image in $G$.

If $\mathbf{B}$ is a picture over $\mathcal{Q}, \partial \mathbf{B}$ denotes the word in $F$ spelled by the arcs traversed along the topological boundary of $\mathbf{B}$. If no arc meets the topological boundary, then $\mathbf{B}$ is called a spherical picture, and its boundary label is $1 \in F$. Two pictures are equivalent if one can be transformed to the other by a sequence of allowable moves. These moves are of three types: insertion/deletion of a floating arc, bridge move, and insertion/deletion of a folding pair. (See [5], [13], for details.) We multiply two pictures by forming their disjoint union, and we invert a picture by taking its mirror image while changing the sign of each disk label. With these operations the equivalence classes of pictures form a group. The normal subgroup generated by spherical pictures is abelian. We write $\mathbf{P}+\mathbf{Q}$ for the product of two spherical pictures, and $\mathbf{B}$ - $\mathbf{D}$ for the product of two arbitrary pictures. Similarly, $\mathbf{- P}$ denotes the inverse of a spherical picture $\mathbf{P}$, while $\mathbf{B}^{-1}$ denotes the inverse of a disk picture $\mathbf{B}$.

If $\mathbf{P}$ is a spherical picture over $\mathcal{Q}$ we let $[\mathbf{P}]$ denote the element of $\pi_{2} Y$ it represents. The left $\mathbf{Z H}$-module structure of $\pi_{2} Y$ is induced by the following $F$-action on spherical pictures. For $w \in F, w \cdot \mathbf{P}$ is the spherical picture obtained by encircling $\mathbf{P}$ with arcs whose labels spell $w$. Then we have the well-defined $H$-action $\bar{w} \cdot[\mathbf{P}]=[w \cdot \mathbf{P}]$, where $\bar{w}$ is the image of $w$ in $H$.

Suppose $C_{*}(\tilde{Y})$ is the chain complex of the universal cover $\tilde{Y}$ of $Y$. The standard $\mathbf{Z} H$-module injection $\mu: \pi_{2} Y \rightarrow C_{2}(\tilde{Y})$ can be described in terms of spherical pictures as follows. Suppose $\mathbf{P}$ over $\mathcal{Q}$ represents $[\mathbf{P}] \in \pi_{2} Y$, and that $\mathbf{P}$ has $k$ disks, $\Delta_{1}, \Delta_{2}, \cdots, \Delta_{k}$, with disk $\Delta_{i}$ getting the label $\omega\left(\Delta_{i}\right)^{\epsilon_{i}}\left(\epsilon_{i}= \pm 1\right.$, and $\omega\left(\Delta_{i}\right)$ is a relator of $\mathcal{Q}$ ) for $i=1,2, \cdots, k$. A transverse path $\gamma_{i}$ from the global basepoint of $\mathbf{P}$ to the basepoint of $\Delta_{i}$ determines a word $\omega\left(\gamma_{i}\right)$ from the arcs it traverses. Let $h_{i}$ be the image of this word in the fundamental group $H$. Then 


$$
\mu([\mathbf{P}])=\sum_{i=1}^{k} \epsilon_{i} h_{i} c_{\omega\left(\Delta_{i}\right)}^{2} .
$$

3. The $\mathbf{N}$-identity property for $(\mathbf{Y}, \mathbf{X})$. A spherical picture over $\mathcal{Q}=\langle\mathbf{x}: \mathbf{r}, \mathbf{s}\rangle$ can have some disks labeled by relators in $\mathbf{r}$, and some with labels from $\mathbf{s}$. We will call the former disks r-disks, and the latter disks s-disks. Let $N<H$ be any subgroup of $H$, and suppose $N_{F}$ is the pre-image of $N$ in the free group $F(\mathbf{x})$.

Definition 3.1. The pair $(Y, X)$ (or $(\mathcal{Q}, \mathcal{P}))$ has the right (resp. left) $N$-identity property if every spherical picture over $\mathcal{Q}$ has a pairing of its s-disks $(i \leftrightarrow j)$ such that

$$
\begin{aligned}
& \omega\left(\Delta_{i}\right)=\omega\left(\Delta_{j}\right) ; \\
& \epsilon_{i} \neq \epsilon_{j} ; \\
& N_{F} \omega\left(\gamma_{i}\right)=N_{F} \omega\left(\gamma_{j}\right)\left(\text { resp. } \omega\left(\gamma_{i}\right) N_{F}=\omega\left(\gamma_{j}\right) N_{F}\right) .
\end{aligned}
$$

Implicit in the definition is a set of transverse paths $\left\{\gamma_{i}\right\}$ to the disk basepoints of disks with labels in s. If the definition is satisfied for a particular set of transverse paths then it is satisfied for any such set. This is a consequence of van Kampen's lemma.

If $\mathbf{r}$ is empty (i.e., if $X$ is the 1-skeleton of $Y$ ), then this definition matches the defintion given in [7] of the right $N$-identity property for the two-complex $Y$.

If $N \triangleleft H$ is normal, then the left and right identity properties coincide. In this case, we will refer to the $N$-identity property. If $(Y, X)$ has the $\{1\}$-identity property, then we say that $(Y, X)$ has the identity property. It is this identity property that appreared in [8]. If $X=Y^{(1)}$, it is well known that the pair $\left(Y, Y^{(1)}\right)$ has the identity property if and only if the 2-complex $Y$ is aspherical.

Note that if the inclusion induced map $i_{\#}: \pi_{2} X \rightarrow \pi_{2} Y$ is surjective, then $(Y, X)$ has the identity property, for if this map is surjective, then any spherical picture over $\mathcal{Q}$ is equivalent to one without $\mathbf{s}$-disks. By virtue of the allowable moves on pictures, it follows that any picture over $\mathcal{Q}$ has the requisite pairing of its $\mathbf{s}$-disks. Thus, for instance, if $Y$ is aspherical and $X$ is any subcomplex containing $Y^{(1)}$, then $(Y, X)$ has the identity property.

For a specific example, consider $\mathcal{P}=\langle a, b:[a, b]\rangle$ and $\mathbf{s}=\left\{a^{3}\right\}$, so that $\mathcal{Q}=$ $\left\langle a, b:[a, b], a^{3}\right\rangle$. Then $(Y, X)$ has the $H$-identity property. Indeed, in the case $N=H$, the third condition in the definition is superfluous. Thus, $(\mathcal{Q}, \mathcal{P})$ has the $H$-identity property if and only if the s-disks of any spherical picture $\mathbf{P}$ over $\mathcal{Q}$ can be paired so that disks in each pair have the same label but opposite orientation. We will say such a picture has parity in $\mathbf{s}$. In view of the well-known $\mathbf{Z} H$-module generators of $\pi_{2} Y$ depicted in Figure 1, it follows that every spherical picture over $\mathcal{Q}$ has parity in $\mathbf{s}$.

We observe from the definition that if $N_{1} \subset N_{2}$ and $(\mathcal{Q}, \mathcal{P})$ has the right $N_{1}$ identity property, then $(\mathcal{Q}, \mathcal{P})$ has the right $N_{2}$-identity property as well.

Let $p: \tilde{Y} \rightarrow Y$ denote the universal covering of $Y$, and consider $p^{-1}(X)=X_{L}$, the covering of $X$ associated to the normal subgroup $L=\langle\langle\mathbf{s}\rangle\rangle_{G}$. The homology sequence of the pair $\left(\tilde{Y}, X_{L}\right)$ yields the following exact sequence of left $\mathbf{Z} H$-modules:

$$
\begin{aligned}
& H_{2}(\tilde{Y}) \quad H_{2}\left(\tilde{Y}, X_{L}\right) \quad H_{1}\left(X_{L}\right) \\
& \|\quad\| \quad \| \\
& 1 \rightarrow H_{2} X_{L} \stackrel{i}{\rightarrow} \quad \pi_{2} Y \stackrel{j}{\rightarrow} \bigoplus_{s \in \mathbf{s}} \mathbf{Z} H c_{s}^{2} \stackrel{\psi}{\rightarrow} \quad H_{1}(L) \quad \rightarrow \quad 1 .
\end{aligned}
$$



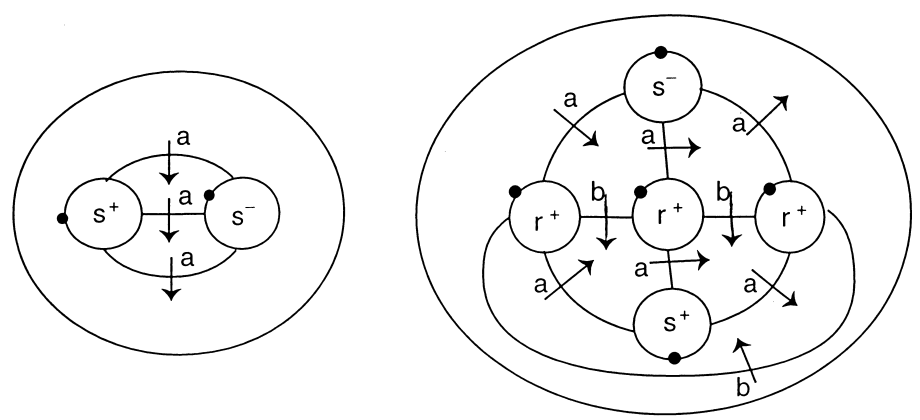

Figure 1

From now on, we will let $\mathbf{Z} H^{\mathbf{s}}$ denote $\bigoplus_{s \in \mathbf{s}} \mathbf{Z} H c_{s}^{2}$. We have a left $H$-action on $H_{1}(L)$, induced by the conjugation action of $G$ on $L$. That is, for $h \in H$, $h \cdot s[L, L]=g \bar{s} g^{-1}[L, L]$, where $g \in G$ has image $h \in H$, and $\bar{s}$ is the image of $s$ in $G$. This action makes $H_{1}(L)$ into a left $\mathbf{Z} H$-module, called the relative relation module associated to $(\mathcal{Q}, \mathcal{P})$.

The maps in (1) are as follows. For $s \in \mathbf{s}, \psi\left(c_{s}^{2}\right)=s[L, L]$. The map $j: \pi_{2} Y \rightarrow \mathbf{Z} H^{\mathbf{s}}$ is the composition

$$
j=\rho \circ \mu: \pi_{2} Y \rightarrow C_{2}(\tilde{Y})=\mathbf{Z} H^{\mathbf{r}} \oplus \mathbf{Z} H^{\mathbf{s}} \rightarrow \mathbf{Z} H^{\mathbf{s}}
$$

where $\rho$ is projection onto the s-coordinates, and $\mu$ is the map defined in the previous section.

For the third map in (1) note that if $\alpha \in \mathbf{Z} H^{\mathbf{r}}$ has image in $H_{2} X_{L}$, then $(\alpha, 0) \in$ $\mathbf{Z} H^{\mathbf{r}} \oplus \mathbf{Z} H^{\mathbf{s}}$ has image in $H_{2}(\tilde{Y})$. Let $i(\alpha)=\left[\mathbf{P}_{\alpha}\right]$, where the spherical picture $\mathbf{P}_{\alpha}$ is a representative of the unique class $\left[\mathbf{P}_{\alpha}\right]$ in $\pi_{2} Y$ with $\mu\left(\left[\mathbf{P}_{\alpha}\right]\right)=[(\alpha, 0)]$.

Given the subgroup $N<H=\pi_{1} Y$, let $Y_{N}$ denote the cover of $Y$ associated to $N$. Let $N_{G}=\iota_{\#}^{-1}(N)$ be the pre-image of $N$ in $G$, and build $X_{N_{G}}$, the cover of $X$ with respect to $N_{G}$. Then $X_{N_{G}}$ is a subcomplex of $Y_{N}$, and the pair $\left(Y_{N}, X_{N_{G}}\right)$ covers $(Y, X)$.

Definition 3.2. Let $N<\pi_{1} Y$. The pair $(Y, X)$ is $N$-Cockcroft if the composite map

$$
\pi_{2}(Y) \stackrel{j}{\rightarrow} \mathbf{Z} H^{\mathbf{s}}=H_{2}\left(\tilde{Y}, X_{L}\right) \stackrel{\rho_{N}}{\rightarrow} H_{2}\left(Y_{N}, X_{N_{G}}\right)
$$

is trivial, where $\rho_{N}$ is the induced map from the projection $\left(\tilde{Y}, X_{L}\right) \rightarrow\left(Y_{N}, X_{N_{G}}\right)$ of covers.

As with the identity property, we recover the $N$-Cockcroft property for a 2 complex $Y$ from the $N$-Cockcroft property for the pair $\left(Y, Y^{(1)}\right)$.

For any group $N$, the augmentation ideal $I N=\operatorname{ker}(\epsilon: \mathbf{Z N} \rightarrow \mathbf{Z})$, where $\epsilon\left(\sum n_{i} h_{i}\right)=\sum n_{i}, n_{i} \in \mathbf{Z}, h_{i} \in N$. Then $I N \cdot \mathbf{Z} H$ is the right ideal of $\mathbf{Z} H$ consisting of all finite sums $\sum a_{i} b_{i}, \quad\left(a_{i} \in I N, b_{i} \in \mathbf{Z} H\right)$.

We may identify the quotient module $\mathbf{Z H} / I N \cdot \mathbf{Z} H$ with $\mathbf{Z} \otimes_{N} \mathbf{Z} H$ by the natural isomorphism $\bar{b} \mapsto 1 \otimes b$ where $\bar{b}$ denotes the image in $\mathbf{Z} H / I N \cdot \mathbf{Z} H$ of an element $b$ in $\mathbf{Z H}$ (see [6, p. 34]). Also, one can check that $\mathbf{Z} H / I N \cdot \mathbf{Z} H \cong \mathbf{Z}[N \backslash H]$ where $N \backslash H$ denotes the set of right cosets of $N$, and that $\mathbf{Z}[N \backslash H] \cong \mathbf{Z}\left[N_{F} \backslash F\right]$ are naturally identified. 
Proposition 3.3. The following statements are equivalent for the pair $(Y, X)$.

1. $(Y, X)$ has the right $N$-identity property.

2. The s-coefficients of any spherical picture $\mathbf{P}$ over $\mathcal{Q}$ lie in the right ideal $I N \cdot \mathbf{Z H}$.

3. $(Y, X)$ is $N$-Cockcroft.

We remark that this proposition is an immediate generalization of parts of Theorem 4.2 in [7], and the proof of Theorem 4.2 may be adapted to this more general setting.

Proof. (1) $\Rightarrow$ (2) Let $\mathbf{P}$ over $\mathcal{Q}$ be an arbitrary spherical picture. For $s \in \mathbf{s}$, let $b_{s}$ denote the coefficient of $c_{s}^{2}$ in $j([\mathbf{P}]) \in \mathbf{Z} H^{s}$. We must show that $b_{s} \in I N \cdot \mathbf{Z} H$. Suppose $\gamma$ is a set of transverse paths to the disk basepoints of $\mathbf{P}$. As usual, let $\omega\left(\gamma_{i}\right)$ be the word in $F$ determined by the path $\gamma_{i}$ to the basepoint of $\Delta_{i}$, and $h_{i}$ this word's image in $\mathrm{H}$. Then $b_{s}=\sum \epsilon_{k} h_{k}$, where the sum runs over all disks $\Delta_{k}$ labelled by $s^{\epsilon_{k}}\left(\epsilon_{k}= \pm 1\right)$. If $(\mathcal{Q}, \mathcal{P})$ has the right $N$-identity property then there exists a pairing of the $s$-disks $\left(i \leftrightarrow j\right.$ ) such that $\epsilon_{i}=-\epsilon_{j}$ and $h_{i}=n_{j} h_{j}$ for some $n_{j} \in N$. We may then rewrite $b_{s}$ as $\sum \epsilon_{j}\left(1-n_{j}\right) h_{j}$ where the sum includes one term for each pair of disks labelled by $s$. Thus, $b_{s} \in I N \cdot \mathbf{Z} H$.

(2) $\Rightarrow$ (1) We can tensor (1) by $\mathbf{Z} \otimes_{\mathbf{Z} N}$ - to obtain the next exact sequence

$$
\mathbf{Z} \otimes_{N} H_{2} X_{L} \stackrel{1 \otimes i}{\rightarrow} \mathbf{Z} \otimes_{N} \pi_{2} Y \stackrel{1 \otimes j}{\rightarrow} \mathbf{Z} \otimes_{N} \mathbf{Z} H^{\mathbf{s}} \stackrel{1 \otimes \psi}{\rightarrow} \mathbf{Z} \otimes_{N} H_{1}(L) \rightarrow 1
$$

Since $\mathbf{Z} \otimes_{N} \mathbf{Z} H^{\mathbf{s}} \cong(\mathbf{Z H} / I N \cdot \mathbf{Z} H)^{\mathbf{s}}$, condition (2) implies the map $1 \otimes j$ is trivial, and hence $1 \otimes \psi$ is an isomorphism. But $\mathbf{Z} \otimes_{N} \mathbf{Z} H^{\mathbf{s}} \cong \oplus_{s \in \mathbf{s}}\left(\mathbf{Z}\left[N_{F} \backslash F\right]\right) c_{s}^{2}$, and $\mathbf{Z} \otimes_{N} H_{1}(L) \cong \mathbf{Z} \otimes_{N} L /[L, L] \cong L /\left[N_{G}, L\right]$, so that

$$
\oplus_{s \in \mathbf{s}}\left(\mathbf{Z}\left[N_{F} \backslash F\right]\right) c_{s}^{2} \cong L /\left[N_{G}, L\right] .
$$

This map is given on basis elements by $c_{s}^{2} \mapsto \bar{s}\left[N_{G}, L\right]$.

Now consider $\mathbf{P}$ over $\mathcal{Q}$, and suppose $j([\mathbf{P}])=\sum \epsilon_{i} \bar{b}_{i} c_{s_{i}}^{2}$ where $\epsilon_{i}= \pm 1, b_{i} \in G$, and $\bar{b}_{i}$ is its image in $H$. From sequence $(1), \psi(j[\mathbf{P}])=0$ implies that $\sum \epsilon_{i} b_{i} \bar{s}_{i} b_{i}^{-1}[L, L]=0$ in $H_{1}(L)$, so that $\sum 1 \otimes \epsilon_{i} b_{i} \bar{s}_{i} b_{i}^{-1}[L, L]=0$ in $\mathbf{Z} \otimes_{N} H_{1}(L)$. Condition (2) implies that this element's pre-image in $\oplus_{s \in \mathbf{s}}\left(\mathbf{Z}\left[N_{F} \backslash F\right]\right) c_{s}^{2}$ is trivial. In particular,

$$
\sum_{i} \epsilon_{i}\left(N_{F} f_{i}\right) c_{s_{i}}^{2}=0
$$

where $f_{i}$ is the pre-image of $b_{i}$ in $F$. So, for each $s$, the partial sum $\sum_{s_{i}=s} \epsilon_{i}\left(N_{F} f_{i}\right) c_{s_{i}}^{2}$ is trivial and the pairing sought necessarily exists.

$(2) \Longleftrightarrow(3)$ This equivalence follows from an analysis of the chain complex of the pair $\left(Y_{N}, X_{N_{G}}\right)$. We may identify $C_{2}\left(Y_{N}\right)$ with $\mathbf{Z} \otimes_{N} C_{2}(\bar{Y}) \cong \mathbf{Z} \otimes_{N} \mathbf{Z} H^{\mathrm{r} \cup \mathbf{s}}$. Similarly, $C_{2}\left(X_{N_{G}}\right) \cong \mathbf{Z} \otimes_{N_{G}} \mathbf{Z} G^{\mathbf{r}}$, from which we may identify $H_{2}\left(Y_{N}, X_{N_{G}}\right) \cong C_{2}\left(Y_{N}, X_{N_{G}}\right)$ with $\mathbf{Z} \otimes_{N} \mathbf{Z} H^{\mathbf{s}}$. Thus, $(\mathcal{Q}, \mathcal{P})$ is $N$-Cockcroft if and only if $\pi_{2}(Y) \stackrel{j}{\rightarrow} \mathbf{Z} H^{\mathbf{s}} \rightarrow$ $(\mathbf{Z H} / I N \cdot \mathbf{Z} H)^{\mathbf{s}}$ is trivial; that is, if and only if $j([\mathbf{P}])$ has coefficients living in $I N \cdot \mathbf{Z} H$ for each $\mathbf{P}$ over $\mathcal{Q}$

One checks that the pair $(\mathcal{Q}, \mathcal{P})$ has the left $N$-identity property if and only if the s-coefficients of any spherical picture over $\mathcal{Q}$ live in the left ideal $\mathbf{Z} H \cdot I N$ of $\mathbf{Z H}$. In 
fact, $(\mathcal{Q}, \mathcal{P})$ has the left $N$-identity property if and only if the s-coefficients of each spherical picture $\mathbf{P}$ in a module generating set of $\pi_{2} Y$ live in $\mathbf{Z H} \cdot I N$. We may restrict our attention to a $\pi_{2}$ generating set in this case precisely because $\pi_{2} Y$ is a left $\mathbf{Z H}$ module. See [14] for a discussion of this left/right distinction in the absolute case.

For example, suppose $\mathcal{P}=\langle\mathbf{x}, t:[t, u]\rangle$ where $u \in F(\mathbf{x})$ is non-trivial, and $t$ is a letter not in $\mathbf{x}$. Let $\mathbf{s}=\{t u\}$, so that $\mathcal{Q}=\langle\mathbf{x}, t:[t, u], t u\rangle$. We remark that $\mathcal{Q}$ is Tietze equivalent to $\langle\mathbf{x}: 1\rangle$. Thus, $\mathcal{Q}$ is not Cockcroft, and $H$ is isomorphic to the free group on $\mathbf{x}$.

If $Y$ is the model on $\mathcal{Q}$, then one can check that the spherical picture over $\mathcal{Q}$ in Figure 2 generates $\pi_{2} Y$ as left $\mathbf{Z} H$-module.

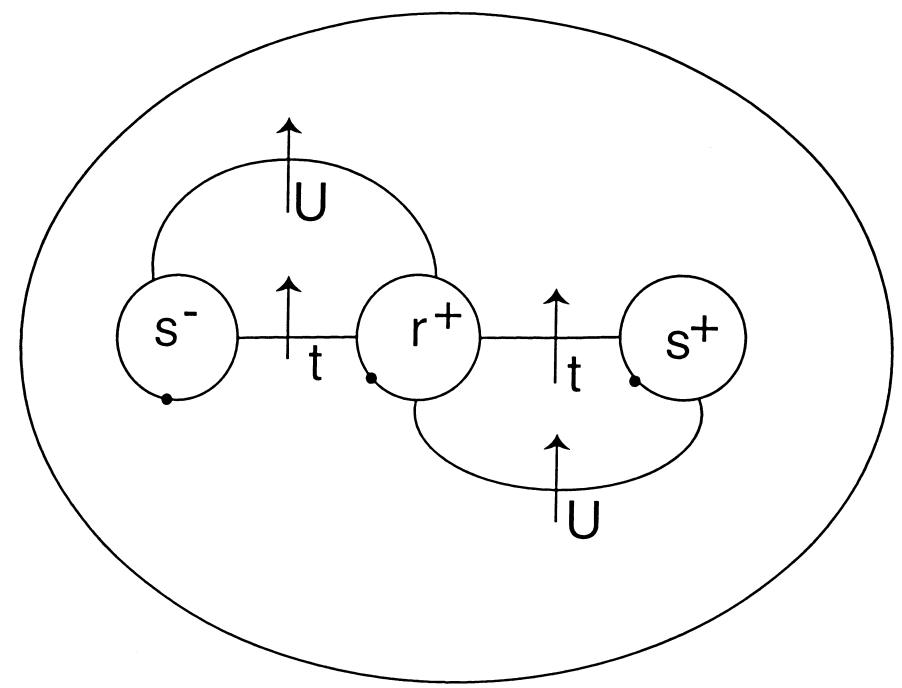

Figure 2

Note that $j([\mathbf{P}])=(\bar{u}-1) c_{s}^{2}$, where $\bar{u}$ is the image of $u$ in $H$, from which we see that $(Y, X)$ has the left $N$-identity property for a subgroup $N<H$ if and only if $\bar{u} \in N$. On the other hand, $(Y, X)$ has the right $N$-identity property if and only if $h(\bar{u}-1) \in I N \cdot \mathbf{Z} H$ for each $h \in H$. Furthermore, $(Y, X)$ is $\langle\langle\bar{u}\rangle\rangle_{H^{-}}$-Cockcroft here, though $Y$ itself is not Cockcroft.

In general, if $N=\{1\}$ then $I N \cdot \mathbf{Z} H$ is trivial. Thus, Proposition 3.3 ensures that $(\mathcal{Q}, \mathcal{P})$ has the identity property if and only if the map $j$ in (1) is trivial. Consider the following diagram with commutative square, obtained from sequence (1) by adding the vertical Hopf sequence:

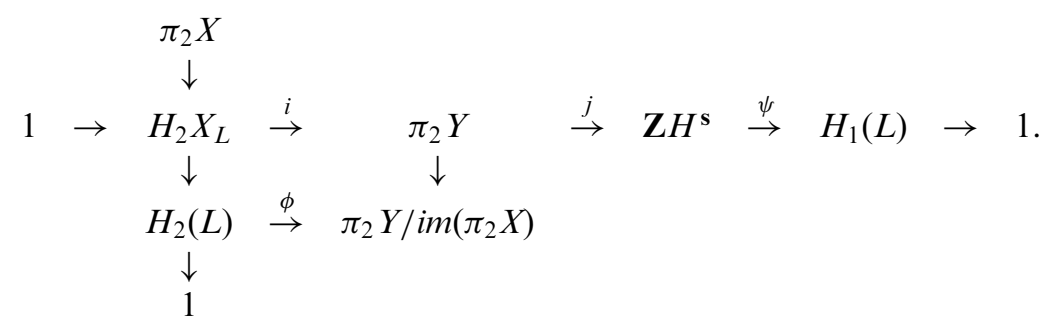


One checks that the map $\phi$ is injective, and that $\phi$ is surjective if and only if $j$ is trivial. Then, the following proposition holds.

Proposition 3.4. The following statements are equivalent.

1. $(Y, X)$ has the identity property.

2. The map $\phi: H_{2}(L) \rightarrow \pi_{2} Y / i m\left(\pi_{2} X\right)$ is a $\mathbf{Z H}$-module isomorphism.

3. The map $\psi: \mathbf{Z} H^{\mathbf{s}} \rightarrow H_{1}(L)$ is a $\mathbf{Z H}$-module isomorphism.

REMARK 3.5. For the reader's convenience, we now consider the strategy for constructing a counterexample to the Whitehead conjecture, as given in [8]. Suppose $X$ is a non-aspherical 2-complex modelled on $\mathcal{P}=\langle\mathbf{x}: \mathbf{r}\rangle$ that is $L_{1}$-Cockcroft, for some non-trivial normal subgroup $L_{1} \triangleleft G=\pi_{1} X$.

Consider a strictly increasing sequence of normal subgroups of $G$

$$
\{1\}=L_{0}<L_{1}<\cdots<L_{n}<L_{n+1}<\cdots<G
$$

and nested sets of elements of $F(\mathbf{x})$

$$
\emptyset=\mathbf{s}_{0} \subset \mathbf{s}_{1} \subset \cdots \mathbf{s}_{n} \subset \mathbf{s}_{n+1} \cdots
$$

such that $L_{n}=\left\langle\left\langle\mathbf{s}_{n}\right\rangle\right\rangle_{G}$. Consider the family of group presentations $\mathcal{P}_{n}=\left\langle\mathbf{x}: \mathbf{r}, \mathbf{s}_{n}\right\rangle$ for each integer $n \geq 0$. ( $\mathcal{P}_{0}=\mathcal{P}$.) Suppose further that for each $n \geq 1$

(i) the pair $\left(\mathcal{P}_{n}, \mathcal{P}\right)$ has the identity property; and

(ii) the map $H_{2} X_{L_{n}} \rightarrow H_{2} X_{L_{n+1}}$ induced by the injection $L_{n} \rightarrow L_{n+1}$ is trivial.

Having such a sequence of presentations, we can construct an infinite counterexample to the Whitehead conjecture as follows.

Let $X_{n}=X \cup\left\{c_{s}^{2}: s \in \mathbf{s}_{n}\right\}$. If $p: \tilde{X}_{n} \rightarrow X_{n}$ is the universal cover, then $p^{-1}(X)=X_{L_{n}}$.

Since $\left(\mathcal{P}_{n}, \mathcal{P}\right)$ has the identity property, $H_{2} X_{L_{n}} \cong \pi_{2} X_{n}$ for each $n$. This fact, condition (ii), and the commutative diagram

$$
\begin{array}{ccc}
H_{2} X_{L_{n}} & \rightarrow & \pi_{2} X_{n} \\
\downarrow & \circ & \downarrow \\
H_{2} X_{L_{n+1}} & \rightarrow & \pi_{2} X_{n+1}
\end{array}
$$

ensure that $\pi_{2} X_{n} \rightarrow \pi_{2} X_{n+1}$ is trivial at each stage. In this way, we build $X_{\infty}=\bigcup X_{n}$, an aspherical 2-complex having the non-aspherical subcomplex, $X$.

4. A group-theoretic gharacterization. In this section we require our subgroup $N \triangleleft H$ to be normal. Suppose $\mathbf{P}$ is a spherical picture over $\mathcal{Q}$. We may focus directly upon the s-disks of $\mathbf{P}$ as follows: consider a set of paths $\left\{\gamma_{i}\right\}$ to the $\mathbf{r}$-disks of $\mathbf{P}$ (see Figure 3). We assume that no two paths intersect except at the global basepoint. In [13] such a set of paths is called a spray, except that here we're restricting a spray to the r-disks. Next, cut along the boundary of this spray to obtain a disk picture having all the s-disks of $\mathbf{P}$ (if any), and boundary label reading

$$
\prod \omega\left(\gamma_{i}\right) \omega\left(\Delta_{i}\right)^{\epsilon_{i}} \omega\left(\gamma_{i}\right)^{-1}
$$


where the product runs over all $\mathbf{r}$-disks $\Delta_{i}$, and $\omega\left(\Delta_{i}\right)^{\epsilon_{i}} \in \mathbf{r} \cup \mathbf{r}^{-1}$ is the label of $\Delta_{i}$. In other words, we obtain a picture over the presentation $\mathcal{Z}=\langle\mathbf{x}: \mathbf{s}\rangle$ whose boundary is in $R$. This leads to the following notion.
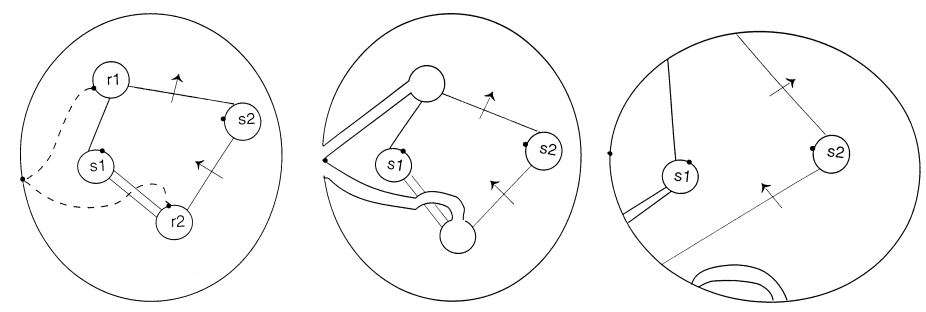

Figure 3

Definition 4.1. Suppose $\mathcal{P}=\langle\mathbf{x}: \mathbf{r}\rangle$ presents the group $G, N \triangleleft G$ is a normal subgroup, and $\mathbf{w}$ is a set of words in $F=F(\mathbf{x})$. Let $N_{F}$ be the pre-image of $N$ in $F$, and $R=\langle\langle r\rangle\rangle_{F}$. Then $\mathcal{P}$ is $N$-Cockcroft ( rel $\mathbf{w}$ ) if and only if any disk picture over $\mathcal{P}$ with boundary label in $W=\langle\langle\mathbf{w}\rangle\rangle_{F}$ has a pairing $(i \leftrightarrow j)$ of its disks such that

$$
\omega\left(\Delta_{i}\right)=\omega\left(\Delta_{j}\right), \quad \epsilon_{i} \neq \epsilon_{j}, \text { and } \omega\left(\gamma_{i}\right) \omega\left(\gamma_{j}\right)^{-1} \in N_{F} .
$$

We say that $\mathcal{P}$ is Cockcroft ( $\mathrm{rel} \mathbf{w}$ ) if $\mathcal{P}$ is $G$-Cockcroft (rel w). We remark that a notion very similar to this (in the case $N=G$ ) was introduced in [2] to study Cockcroft properties of pictures arising from group constructions. The following key lemma is a generalization of a fact stated in [2] for the case $N=G$.

Lemma 4.2. With the notation as in 4.1, $\mathcal{P}$ is $N$-Cockcroft (rel $\mathbf{w}$ ) if and only if $\mathcal{P}$ is $N$-Cockcroft and $W \cap R \subset\left[R, N_{F}\right]$.

Proof. First, suppose $\mathcal{P}$ is $N$-Cockcroft (rel w). Since $1 \in W$ it follows that $\mathcal{P}$ has the $N$-identity property; that is, all spherical pictures over $\mathcal{P}$ have the appropriate pairing of its disks. Thus, $\mathcal{P}$ is $N$-Cockcroft. We must show that $W \cap R \subset\left[R, N_{F}\right]$.

Consider $v \in W \cap R$. Since $v \in R$, van Kampen's Lemma guarantees a picture $\mathbf{B}$ over $\mathcal{P}$ having $v$ as its boundary label. Since $v \in W$, the disks of $\mathbf{B}$ have the prescribed pairing of Definition 4.1. We must show this forces $\partial \mathbf{B}=v \in\left[R, N_{F}\right]$.

Consider any spray of paths $\gamma=\left\{\gamma_{1}, \cdots, \gamma_{k}\right\}$ to the disk basepoints in $\mathbf{B}$, where $\gamma_{i}$ attaches to disk $\Delta_{i}$. It is well known (see [13]) that the word determined by this spray, $\prod \omega\left(\gamma_{i}\right) \omega\left(\Delta_{i}\right)^{\epsilon_{i}} \omega\left(\gamma_{i}\right)^{-1}$, is freely equal to the boundary label of the picture $\mathbf{B}$. That is,

$$
\partial \mathbf{B}=\prod \omega\left(\gamma_{i}\right) \omega\left(\Delta_{i}\right)^{\epsilon_{i}} \omega\left(\gamma_{i}\right)^{-1}
$$

But the words in this product are paired according to Definition 4.1. Armed with this pairing, one checks that the product is trivial in $F /\left[R, N_{F}\right]$. (An easy way to see this is to choose a spray of paths in $\mathbf{B}$ so that paired disks are adjacent in the (clockwise) sequence of paths comprising the spray.) 
Conversely, consider a picture $\mathbf{B}$ over $\mathcal{P}$ having boundary label $\partial \mathbf{B} \in W$. We show that $\mathbf{B}$ has the prescribed pairing. By van Kampen's Lemma, $\partial \mathbf{B} \in R$, and so $\partial \mathbf{B} \in W \cap R \subset\left[R, N_{F}\right]$. It follows that $\partial \mathbf{B}$ is freely equal to a word $v$ of the form

$$
v=\prod_{i=1}^{k} f_{i}\left[w_{i}, u_{i}\right] f_{i}^{-1}
$$

where each $f_{i} \in F$, each $u_{i} \in N_{F}$ and each $w_{i} \in R$.

Now, consider the picture $\mathbf{D}$ in Figure 4 having boundary label equal to $v$.

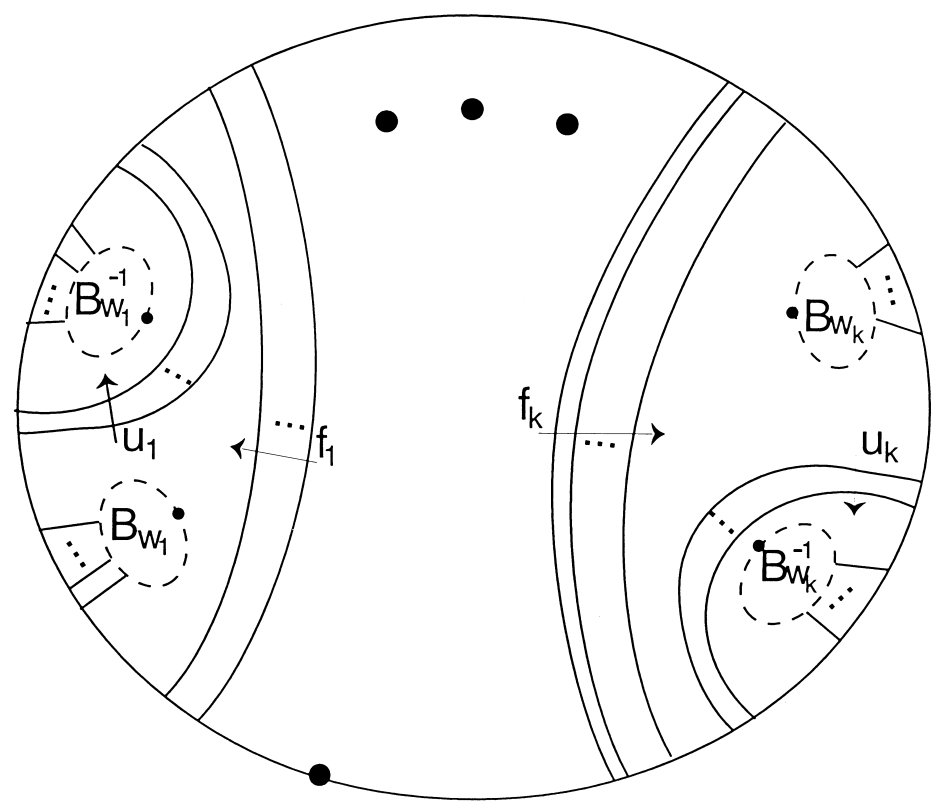

Figure 4

Each subpicture $\mathbf{B}_{w_{i}}$ of $\mathbf{D}$ is a picture over $\mathcal{P}$ associated to $w_{i} \in R$ (this picture has boundary label identitically equal to $w_{i}$ ). Notice that by pairing each disk of $\mathbf{B}_{w_{i}}$ with its mirror image in $\mathbf{B}_{w_{i}}^{-1}$, the disks of $\mathbf{D}$ can be paired to satisfy the conditions of Definition 4.1. Indeed, we may always choose a path from a disk of $\mathbf{B}_{w_{i}}$ to its mirror image in $\mathbf{B}_{w_{i}}^{-1}$ whose associated word is a conjugate of $u_{i} \in N_{F}$, and hence in $N_{F}$, since $N$ is normal.

Let $\mathbf{Q}$ be the spherical picture associated to $\mathbf{B} \cdot \mathbf{D}^{-1}$. Since $\mathcal{P}$ is $N$-Cockcroft, $\mathbf{Q}$ has an appropriate pairing of its disks. Now the disks of $\mathbf{D}$ may be paired appropriately as indicated above, so the subpicture $\mathbf{B}$ has a pairing of its disks as well.

Recall, $Z$ is the 2-complex modelled on $\mathcal{Z}, A=\pi_{1} Z$, and set $M=\langle\langle r\rangle\rangle_{A}$. Let $\pi_{A}: A \rightarrow H$ and $\pi_{F}: F \rightarrow H$ denote the inclusion induced maps on the fundamental groups $\pi_{1} Z \rightarrow \pi_{1} Y$ and $\pi_{1} Y^{(1)} \rightarrow \pi_{1} Y$, respectively.

Theorem 4.3. Suppose $N$ is normal subgroup of $H$, and let $N_{A}=\pi_{A}^{-1}(N)$ and $N_{F}=\pi_{F}^{-1}(N)$. The following statements are equivalent. 
1. $(\mathcal{Q}, \mathcal{P})$ has the $N$-identity property;

2. $\mathcal{Z}$ is $N_{A}$-Cockcroft (rel $\mathbf{r}$ ); and

3. $\mathcal{Z}$ is $N_{A}$-Cockcroft and $R \cap S \subset\left[S, N_{F}\right]$.

Proof. (1) $\Rightarrow(2)$ Suppose $\mathbf{B}$ is a picture over $\mathcal{Z}$ with boundary label in $R$. Then there is a disk picture $\mathbf{D}$ over $\mathcal{P}$ with boundary label identically equal to $\partial \mathbf{B}$ by van Kampen's lemma. Then B. $\mathbf{D}^{-1}$ is equivalent to a spherical picture over $\mathcal{Q}$, and since $(\mathcal{Q}, \mathcal{P})$ has the $N$-identity property, this spherical picture has the appropriate pairing of its s-disks. Thus, the subpicture $\mathbf{B}$, which contains all the s-disks of $\mathbf{B} \cdot \mathbf{D}^{-1}$ must have a pairing of its s-disks satisfying the conditions of Definition 4.1. Indeed the pairing that works for the spherical picture $\mathbf{B} \cdot \mathbf{D}^{-1}$ restricts to a suitable pairing for B.

$(2) \Rightarrow(1)$ : Suppose $\mathbf{P}$ is a spherical picture over $\mathcal{Q}$. We may cut the $\mathbf{r}$-disks from $\mathbf{P}$ as in Figure 3 to form a disk picture over $\mathcal{Z}$ with boundary in $R$. (If $\mathbf{P}$ has no $\mathbf{r}$-disks, then the original picture is unchanged.) This new picture has a prescribed pairing by assumption. Moreover, this pairing can be used in $\mathbf{P}$ to see that the original picture $\mathbf{P}$ has an appropriate pairing.

$(2) \Longleftrightarrow(3)$ : This follows as a corollary to the above lemma, since the pre-image of $N_{A}$ in the free group $F$ is $N_{F}$.

COROLLARY 4.4. The following statements are equivalent.

1. $(\mathcal{Q}, \mathcal{P})$ has the identity property;

2. $\mathcal{Z}$ is $M$-Cockcroft (rel $\mathbf{r}$ ); and

3. $\mathcal{Z}$ is $M$-Cockcroft and $R \cap S \subset[S, R S]$.

Proof. The pre-image of the trivial normal subgroup $\{1\}$ in $A$ is $M$, and the preimage of $M$ in $F$ is $R S$. The corollary is a restatement of Theorem 4.3 in this special case.

EXAmple 4.5. Consider any 2-relator presentation $\mathcal{Z}=\langle\mathbf{x}: u, v\rangle$ where $u, v \in F(\mathbf{x})=F$. Let $\mathcal{P}=\left\langle\mathbf{x}:[u, v]^{n}\right\rangle$ and $\mathcal{Q}=\left\langle\mathbf{x}:[u, v]^{n}, u, v\right\rangle$, for $n \geq 2$. Let $r=[u, v]^{n}, R=\langle\langle r\rangle\rangle_{F}, s_{1}=u, s_{2}=v$ and $S=\left\langle\left\langle\left\{s_{1}, s_{2}\right\}\right\rangle\right\rangle_{F}$. Since $r \in S \cap[S, S]$, it follows that $R \subset S \cap[S, R S]$, and the group-theoretic condition of the corollary holds. So long as $\mathcal{Z}$ is $M$-Cockcroft (e.g., let $\mathcal{Z}$ be any two relator, aspherical presentation), then $(\mathcal{Q}, \mathcal{P})$ has the identity property.

We observe further that the inclusion induced map $\pi_{2} X \rightarrow \pi_{2} Y$ is trivial, while both homotopy groups are non-zero. As the model of a one relator presentation, $\pi_{2} X$ is generated as a $\mathbf{Z} G$-module by a complete dipole. However, this dipole dissolves in the presence of the new s-disks. To see this, note that the root $[u, v]$ of the relator $r$ is contained in $S$. It follows that $X$ is $L$-Cockcroft. Thus $\pi_{2} X \rightarrow \pi_{2} Y$ is trivial. Finally, $\pi_{2} Y$ is not trivial. In the case $n=2$, a non trivial spherical picture over $\mathcal{Q}$ is given in Figure 5. We remark that this picture also demonstrates that $Y$ itself is not Cockcroft.

In [8], Dyer's strategy for constructing a counterexample of infinite type to Whitehead's Conjecture is to begin with a 2-complex pair $(Y, X)$ for which $(Y, X)$ has the identity property and $0 \neq \pi_{2} X \rightarrow \pi_{2} Y$ is trivial. To be able to extend this pair to a longer chain of suitable 2-complexes, it is necessary for $Y$ to be Cockcroft. That is, if $Y$ is not Cockcroft then there exists no 2-complex $Y^{\prime}$ containing $Y$ for which the inclusion induced map $\pi_{2}(i): \pi_{2} Y \rightarrow \pi_{2} Y^{\prime}$ is trivial. We have seen that the 


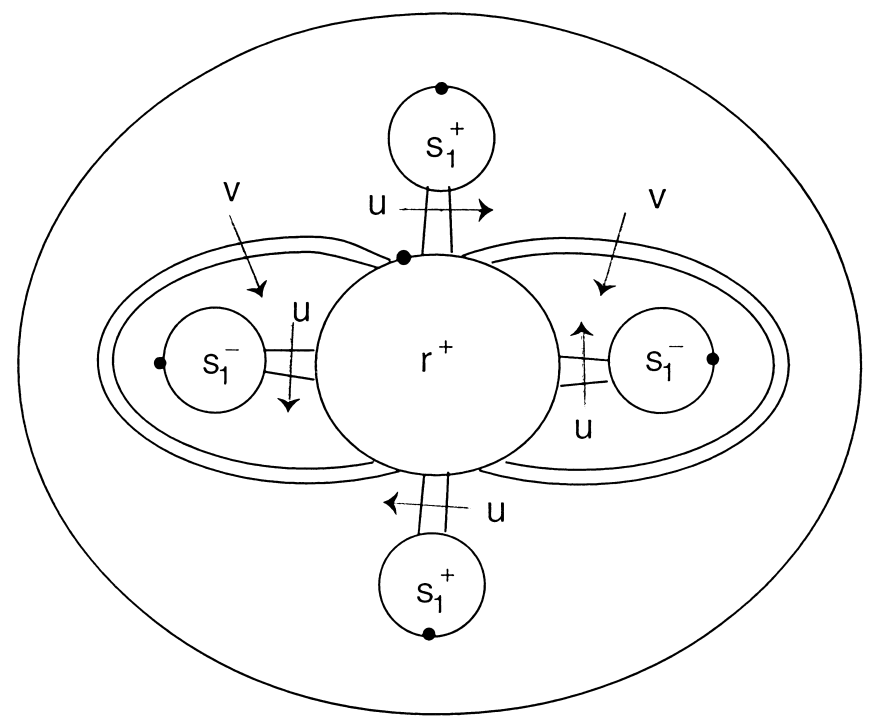

Figure 5

above example satisfies the first two conditions, but is not delicate enough to satisfy the third. It would be of considerable interest to find non trivial examples (where $\pi_{2} X \neq 0$ ) of presentations that satisfy the three conditions of the following corollary.

COROLlary 4.6. The following sets of conditions are equivalent.

$$
\left\{\begin{array}{c}
\pi_{2} X \stackrel{0}{\rightarrow} \pi_{2} Y \\
(Y, X) \text { has the identity property } \\
Y \text { is Cockcroft }
\end{array}\right\} \Leftrightarrow\left\{\begin{array}{c}
X \text { is } L-\text { Cockcroft } \\
Z \text { is } M-\text { Cockcroft } \\
R \cap S \subset[S, R S] \cap[R, F]
\end{array}\right\} .
$$

Proof. For $(\mathcal{Q}, \mathcal{P})$ to have the identity property and $Q$ to be Cockcroft, we must have $j([\mathbf{P}])=0$ for all spherical pictures over $\mathcal{Q}$ and all these pictures must have parity in $r$, for all $r \in \mathbf{r}$. That is, $\mathcal{Z}$ must be $M$-Cockcroft (rel $\mathbf{r}$ ) and $\mathcal{P}$ must be $G$ Cockcroft (rel s). The result now follows from Corollary 4.4 and Lemma 4.2.

5. Combinatorial asphericity. Certain relations always hold in the relative relation module $H_{1}(L)$. Let $C_{G}(s)$ denote the centralizer of the image of the word $s$ in $G$. (For each $s \in \mathbf{s}$ we let $s$ also denote this word's image in the group $G$; context will make clear whether we're viewing $s \in F$ or $s \in G$.)

For any $g \in C_{G}(s)$, let $\bar{g}$ be its image in $H$. Then

$$
(\bar{g}-1) \cdot s[L, L]=g s g^{-1}[L, L]-s[L, L]=s[L, L]-s[L, L]=0 .
$$

We call the set $\left\{(\bar{g}-1) \cdot s[L, L]: s \in \mathbf{s}, g \in C_{G}(s)\right\}$ the set of trivial identities in the relative relation module $H_{1}(L)$, and we say $(\mathcal{Q}, \mathcal{P})$ (or $(Y, X)$ ) has the generalized identity property if $H_{1}(L)$ with generators $\{s[L, L]: s \in \mathbf{s}\}$ is defined by the trivial identities. 
The generalized identity property reduces to the identity property in the case that the image of $C_{G}(s)$ in $H$ is trivial for each $s$. We have observed that if $(Y, X)$ has the identity property then $\pi_{2} Y \cong H_{2} X_{L}$. We turn to relative dipoles to study $\pi_{2} Y$ in the presence of the generalized identity property.

Suppose $\mathbf{P}$ is a spherical picture over $\mathcal{Q}$ containing exactly 2 s-disks and possibly some $\mathbf{r}$-disks. Then $\mathbf{P}$ is called a relative $\mathbf{s}$-dipole, or simply an $\mathbf{s}$-dipole, if the two $\mathbf{s}-$ disks are labelled by the same element of $\mathbf{s}$ but with opposite signs. Notice the $\pi_{2}$ generators in Figure 1 are both s-dipoles.

If $\mathcal{B}_{\mathcal{P}}$ is a set of spherical pictures over $\mathcal{P}$ that generates $\pi_{2} X$ as left $\mathbf{Z} G$-module, then a set $\mathcal{B}$ of spherical pictures over $\mathcal{Q}$ generates $\pi_{2} Y$ over $\mathcal{P}$ if $\mathcal{B} \cup \mathcal{B}_{\mathcal{P}}$ generates $\pi_{2} Y$ as left $\mathbf{Z} H$-module. Two spherical pictures $\mathbf{P}$ and $\mathbf{Q}$ over $\mathcal{Q}$ are called equivalent $\left(\right.$ rel $\left.\mathcal{B}_{\mathcal{P}}\right)$ if $[\mathbf{P}]-[\mathbf{Q}] \in \operatorname{im}\left(\iota_{\#}: \pi_{2} X \rightarrow \pi_{2} Y\right)$.

We will call the pair $(\mathcal{Q}, \mathcal{P})$ (or $(Y, X)$ ) combinatorially aspherical, denoted $(\mathrm{CA})$, if $\pi_{2} Y$ is generated over $\mathcal{P}$ by a set of $\mathbf{s}$-dipoles.

Example 5.1. Suppose $\langle\mathbf{x}: \mathbf{s}\rangle$ is a (CA) presenation of an infinite group. We construct a relative (CA) pair as follows. Let $t$ be a letter not in $\mathbf{x}$, and let $u \in F(\mathbf{x})$ be a word having infinite order in $\langle\mathbf{x}: \mathbf{s}\rangle$. Let $w=t u t$ and $r_{x}=[x, w]$ for each $x \in \mathbf{x}$. Finally, set $\mathbf{r}=\left\{r_{x}: x \in \mathbf{x}\right\}$, and consider the pair $\mathcal{P}=\langle\mathbf{x}, t: \mathbf{r}\rangle$ and $\mathcal{Q}=\langle\mathbf{x}, t: \mathbf{r}, \mathbf{s}\rangle$. It follows from work on generalized graphs of groups in [2] (see also [5]) that $\pi_{2} Y$ is generated over $\mathcal{P}$ by $\pi_{2}$ generators of $\langle\mathbf{x}: \mathbf{s}\rangle$ (which are dipoles since this presentation is (CA)) and one additional spherical pictures for each $s \in \mathbf{s}$. In particular, if $s=x_{1}^{\epsilon_{1}} x_{2}^{\epsilon_{2}} \cdots x_{n}^{\epsilon_{n}}$, with each $x_{i} \in \mathbf{x}$, each $\epsilon_{i}= \pm 1$, then the relator $s$ contributes the $\pi_{2}$ generator pictured in Figure 6. Thus, $(\mathcal{Q}, \mathcal{P})$ is $(\mathrm{CA})$.

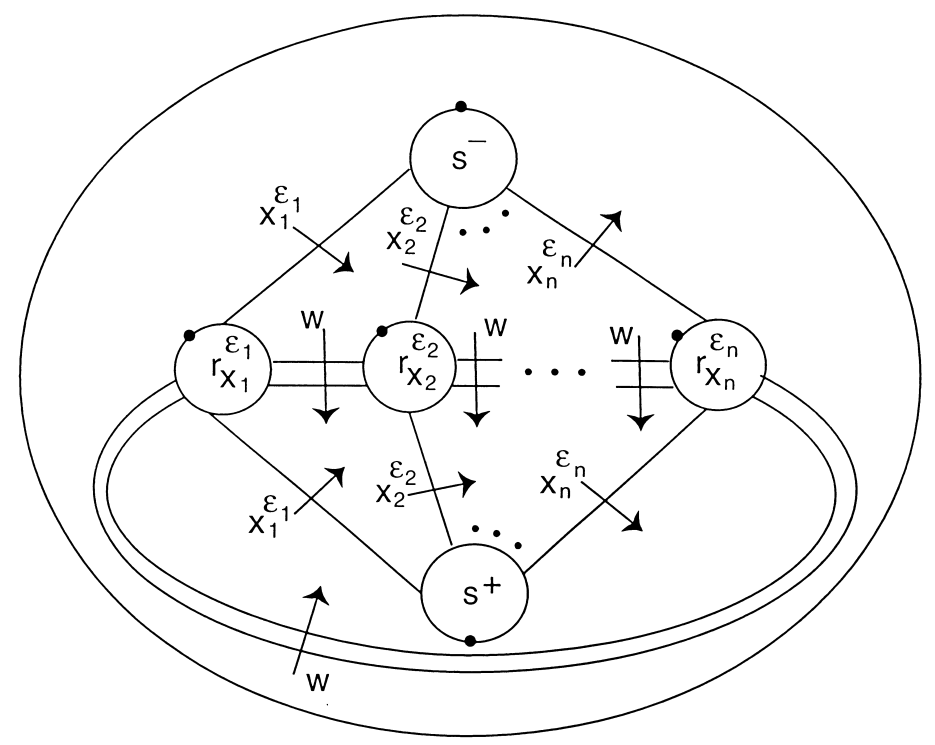

Figure 6

In [2] it is proved that $w$ has infinite order in $H$, and it follows that these examples $(\mathcal{Q}, \mathcal{P})$ do not have the identity property. Thus, in the relative setting (as in the absolute setting), combinatorial asphericity is a weaker notion than the identity property. 
We summarize the above discussion with the following proposition.

Proposition 5.2. Suppose $\langle\mathbf{x}: \mathbf{s}\rangle$ is $(C A)$, and $u \in F(\mathbf{x})$ has infinite order in the group presented. If $\mathcal{P}=\langle\mathbf{x}, t:[x, t u t](x \in \mathbf{x})\rangle$, and $\mathcal{Q}=\langle\mathbf{x}, t:[x, t u t](x \in \mathbf{x}), \mathbf{s}\rangle$, then $(\mathcal{Q}, \mathcal{P})$ is $(C A)$.

For each $1 \neq w \in C_{G}(s)$, for each $s \in \mathbf{s}$, we may construct a basic $\mathbf{s}$-dipole $\mathbf{P}_{w}$ as depicted in Figure 7.

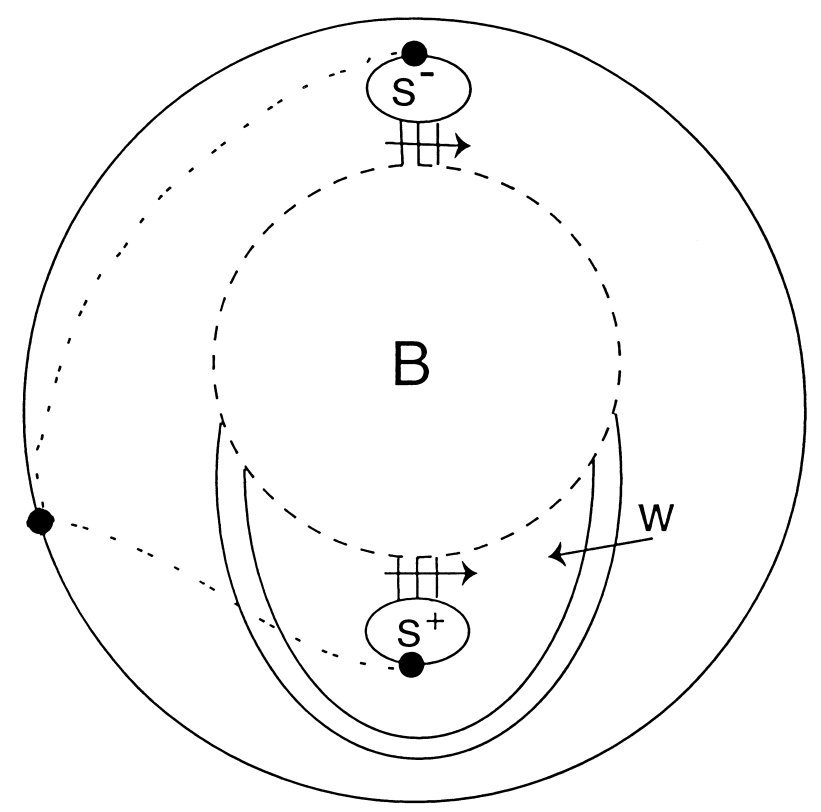

Figure 7

In this construction one may choose the word $W \in F$ representing $w$, and the subpicture $\mathbf{B}$ of $\mathbf{P}_{w}$ having boundary label $W s W^{-1} s^{-1} \in R$ (such a picture $\mathbf{B}$ must exist by van Kampen's Lemma). In general, such choices will lead to inequivalent basic s-dipoles. However $\mathbf{P}_{w}$ is unique modulo $\pi_{2} X$ in the sense described in the following lemma.

Lemma 5.3. Let $\mathcal{B}_{\mathcal{P}}$ be a set of spherical pictures over $\mathcal{P}$ that generates $\pi_{2} X$. Suppose $w \in C_{G}(s)$, for $s \in \mathbf{s}$, and $\mathbf{P}_{w}$ is a basic $\mathbf{s}$-dipole formed from the word $W \in F$ representing $w$ and the picture $\mathbf{B}$ over $\mathcal{P}$ with $\partial \mathbf{B}=[W, s]$. If $\mathbf{P}_{w}^{\prime}$ is a second basic $\mathbf{s}-$ dipoles formed from $W^{\prime} \in F$ representing $w$ and $\mathbf{B}^{\prime}$ with $\partial \mathbf{B}^{\prime}=\left[W^{\prime}, s\right]$, then $\mathbf{P}_{w}$ and $\mathbf{P}_{w}^{\prime}$ are equivalent $\left(\right.$ rel $\left.\mathcal{B}_{\mathcal{P}}\right)$.

Proof. Since $W$ and $W^{\prime}$ both represent the element $w$ of $G$, van Kampen's Lemma ensures the existence of a picture $\mathbf{D}$ over $\mathcal{P}$ with $\partial \mathbf{D}=W^{-1} W^{\prime}$. Now consider $\mathbf{P}_{w}-\mathbf{P}_{w}^{\prime}$ (Figure 8 (a)). Into this picture we may insert the trivial picture $\mathbf{D} \cdot \mathbf{D}^{-1}$ as depicted in Figure 8 (b). Now we make a series of bridge moves to split off the sdisks (Figure 8 (c)) and then fold them from the picture (Figure 8 (d)). That is, $\mathbf{P}_{w}-\mathbf{P}_{w}^{\prime}$ is equivalent to a spherical picture over $\mathcal{P}$. Thus, any two basic s-dipoles associated to $w \in C_{G}(s)$ are equivalent $\left(\operatorname{rel} \mathcal{B}_{\mathcal{P}}\right)$. 

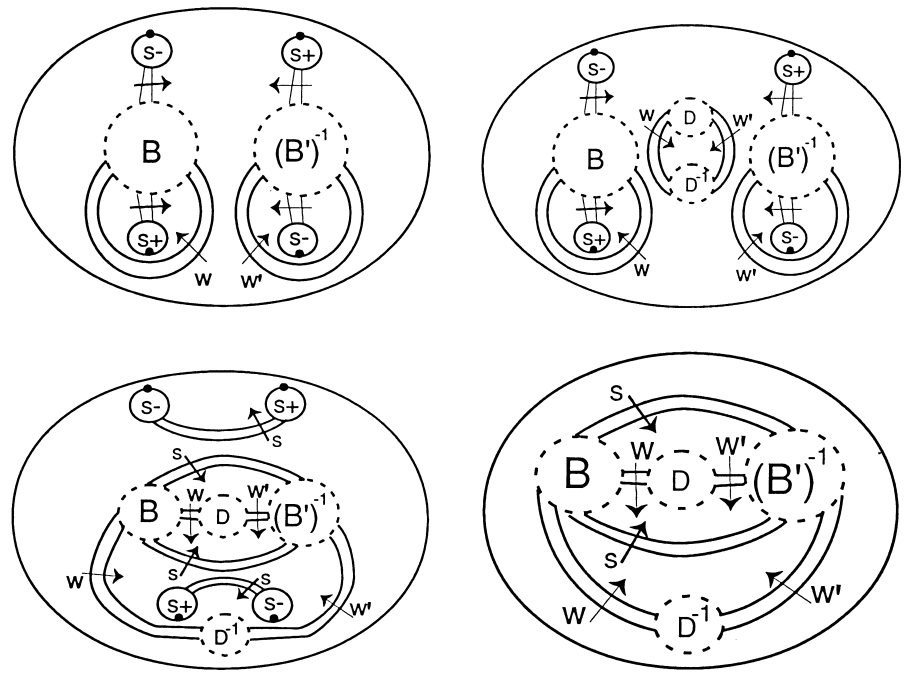

Figure 8

For each $s \in \mathbf{s}$, construct one basic s-dipole $\mathbf{P}_{w}$ for each $w \in C_{G}(s)$. Let $\mathcal{D}_{s}^{*}$ denote this set of basic s-dipoles, and let $\mathcal{D}^{*}=\bigcup_{s \in \mathbf{s}} \mathcal{D}_{s}^{*}$. Finally, let $J\left(\mathcal{D}^{*}\right)$ denote the submodule of $\pi_{2} Y$ generated by $\mathcal{D}^{*}$.

\section{Lemma 5.4. If $\mathbf{P}$ is any $\mathbf{s}$-dipole over $\mathcal{Q}$, then $[\mathbf{P}] \in J\left(\mathcal{D}^{*}\right)$.}

Proof. An arbitrary s-dipole $\mathbf{P}$ contains the local configuration of Figure 9 (a), where we assume a path to the negatively oriented $s$-disk determines the word $V \in F$. Consider the picture $V^{-1} \cdot \mathbf{P}$ depicted in Figure 9 (b).

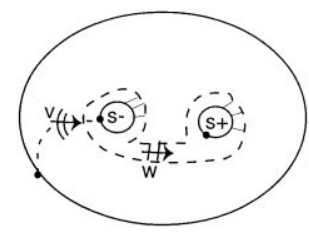

(a)

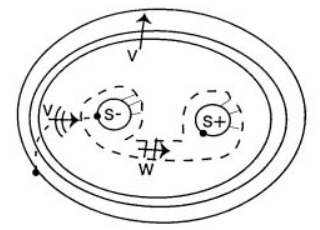

(b)

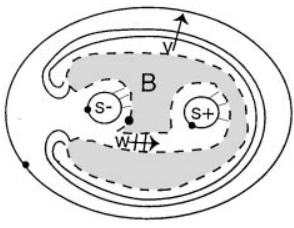

(c)

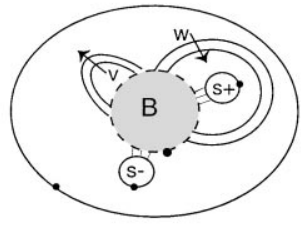

(d)

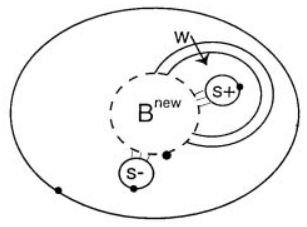

(e)

Figure 9

By bridge moves we may open a path connecting the global basepoint to the basepoint of the negatively oriented disk so that the two basepoints are in the same region. See Figure 9 (c). We may contain all the $\mathbf{r}$-disks of this new picture within the 
shaded subpicture having boundary label from the designated "basepoint" identically equal to $s V^{-1} V W s^{-1} W^{-1}$.

After planar isotopy, we may view our picture as in Figure 9 (d), and upon including the arcs labelled by $V$ into the shaded subpicture we obtain a basic sdipole. By Lemma 5.3, this dipole is equivalent $\left(\right.$ rel $\mathcal{B}_{\mathcal{P}}$ ) to the s-dipole $\mathbf{P}_{w}$ in $\mathcal{D}^{*}$ where $w$ is the image of $W$ in $G$. It follows that the original s-dipole $\mathbf{P}$ is equivalent $\left(\right.$ rel $\mathcal{B}_{\mathcal{P}}$ ) to $V \cdot \mathbf{P}_{w}$, so $[\mathbf{P}] \in J\left(\mathcal{D}^{*}\right)$.

Now suppose that $\mathcal{A}_{s} \subset G$ is a generating set for $C_{G}(s)$, and $\mathcal{D}_{s}=\left\{\mathbf{P}_{w}: w \in \mathcal{A}_{s}\right\}$ for each $s \in \mathbf{s}$. Set $\mathcal{D}=\bigcup_{s \in \mathbf{s}} \mathcal{D}_{s}$ and $J(\mathcal{D})$ to be the submodule of $\pi_{2} Y$ generated by $\mathcal{D}$.

LeMma 5.5. $J(\mathcal{D})=J\left(\mathcal{D}^{*}\right)$.

Proof. Clearly $J(\mathcal{D}) \subset J\left(\mathcal{D}^{*}\right)$. The reverse inclusion follows from two facts. First, for $w \in \mathcal{A}_{s}, \mathbf{P}_{w^{-1}}$ is equivalent to $\left(w^{-1} \cdot \mathbf{P}_{w}\right)^{-1}$. Second, if $w_{1}, w_{2} \in C_{G}(s)$ and $w=w_{1}^{\epsilon_{1}} w_{2}^{\epsilon_{2}}$, then $\mathbf{P}_{w}$ is equivalent to $\left(\mathbf{P}_{w_{1}^{\epsilon_{1}}}\right)+w_{1}^{\epsilon_{1}} \cdot\left(\mathbf{P}_{w_{2}^{\epsilon_{2}}}\right)\left(\right.$ rel $\left.\mathcal{B}_{\mathcal{P}}\right)$. Both equivalences can be checked directly by performing moves on pictures, and the second is demonstrated schematically in Figure 10.

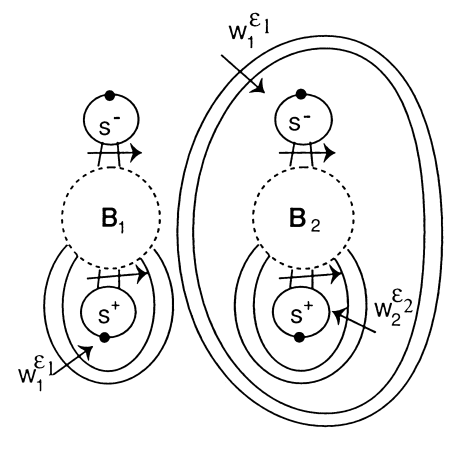

(a)

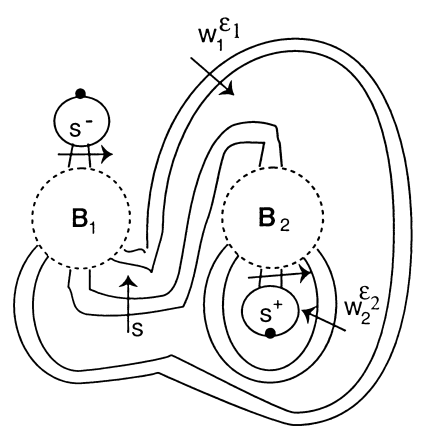

(c)

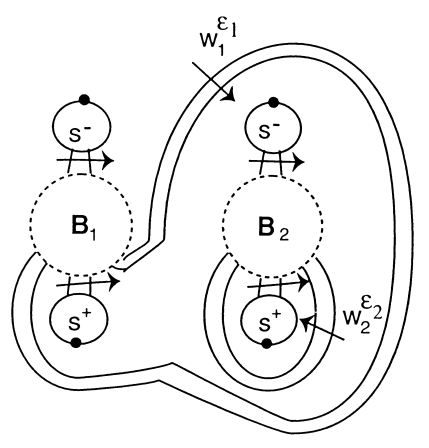

(b)

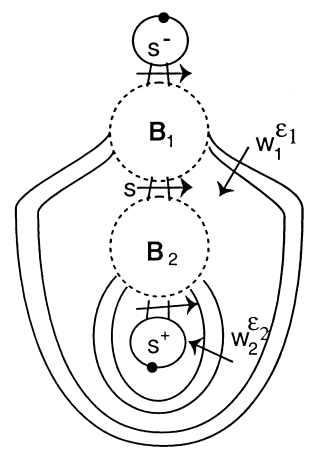

(d)

Figure 10 
Proposition 5.6. $(\mathcal{Q}, \mathcal{P})$ is $(C A)$ if and only if $\pi_{2} Y$ is generated over $\mathcal{P}$ by $\mathcal{D}$.

Proof. If $\pi_{2} Y$ is generated by the set $\mathcal{B}_{\mathcal{P}} \cup \mathcal{D}$, then $(\mathcal{Q}, \mathcal{P})$ is (CA) by definition. Conversely, if $(\mathcal{Q}, \mathcal{P})$ is $(\mathrm{CA})$ then $\pi_{2} Y$ is generated by the set $\mathcal{B}_{\mathcal{P}}$ together with some set of s-dipoles. But the two previous lemmas ensure that any s-dipole in this set is equivalent $\left(\right.$ rel $\mathcal{B}_{\mathcal{P}}$ ) to an element of the submodule of $\pi_{2} Y$ generated by $\mathcal{D}$. The result follows.

We remark that different pictures in $\mathcal{D}$ may be equivalent. For instance, if $\mathcal{P}=\langle a, b:[a, b]\rangle$ and $s=a b^{-1}$, then $C_{G}(s)=G$ is generated by $a$ and $b$. One may check that the basic $s$-dipoles $\mathbf{P}_{a}$ and $\mathbf{P}_{b}$ determined by these words are equivalent. Nonetheless, we can find minimal generating sets in some interesting cases. For the remainder of this paper, we assume the set $\mathcal{D}$ has been chosen, and consists of one basic s-dipole $\mathbf{P}_{w}$ for each $w \in \mathcal{A}_{s}$ in a generating set for $C_{G}(s)$, for each $s \in \mathbf{s}$.

Proposition 5.7. If $(\mathcal{Q}, \mathcal{P})$ is $(C A)$ and $N<H$, then $(\mathcal{Q}, \mathcal{P})$ has the right $N$ identity property if and only if $N$ contains the normal subgroup $K=\left\langle\left\langle\left\{\mathcal{A}_{s}: s \in \mathbf{s}\right\}\right\rangle\right\rangle_{H}$ of $H$.

Proof. If $(\mathcal{Q}, \mathcal{P})$ is $(\mathrm{CA})$ then $\pi_{2} Y$ is generated by the set $\mathcal{B}_{\mathcal{P}} \cup \mathcal{D}$. The image in $j$ of any picture in this set is either trivial or of the form $\left(\bar{w}_{s}-1\right) c_{s}^{2}$ for some $s \in \mathbf{s}, w_{s} \in \mathcal{A}_{s}$. Thus, any picture $\mathbf{P}$ over $\mathcal{Q}$ has image in $j$ of the form

$$
j(\mathbf{P})=\sum h_{i}\left(\bar{w}_{s_{i}}-1\right) c_{s_{i}}^{2},
$$

where $h_{i} \in H, w_{s_{i}} \in \mathcal{A}_{s_{i}}, s_{i} \in \mathbf{s}$. With respect to a subgroup $N<H$, $\mathbf{P}$ has the prescribed pairing of Definition 3.1 if and only if

$$
N_{F} u_{i} w_{s_{i}}=N_{F} u_{i}
$$

where $u_{i} \in F$ represents $h_{i} \in H$. That is, $\mathbf{P}$ has the prescribed pairing if and only if $u_{i} w_{s_{i}} u_{i}^{-1} \in N_{F}$. It follows that all pictures over $\mathcal{Q}$ have the prescribed pairing if and only if $K \subset N$.

For instance, let $\mathcal{P}=\langle a, b:[a, b]\rangle$ and $s=a^{3}$. Then $(\mathcal{Q}, \mathcal{P})$ is $(\mathrm{CA})$ since $\pi_{2} Y$ is generated by s-dipoles (as shown in Figure 1). Since $G$ is abelian in this case, the centralizer $C_{G}(s)=G$ is generated by $a$ and $b$. The normal subgroup of $H$ generated by $a$ and $b$ is $H$ itself, so the above result ensures that $(\mathcal{Q}, \mathcal{P})$ has the right $N$-identity property if and only if $N=H$.

Proposition 5.8. If $(\mathcal{Q}, \mathcal{P})$ is $(C A)$ then $(\mathcal{Q}, \mathcal{P})$ has the generalized identity property.

Proof. According to the exactness of sequence (1), $H_{1}(L)$ is isomorphic to the $\mathbf{Z} H$-module generated by $\left\{c_{s}^{2}: s \in \mathbf{s}\right\}$ and defined by the relations $j([\mathbf{P}])=0$ for all pictures in a generating set of $\pi_{2} Y$. In the present situation, we may assume $\pi_{2} Y$ is generated by the pictures in $\mathcal{B}_{\mathcal{P}} \cup \mathcal{D}$. Since any picture $\mathbf{P}$ over $\mathcal{P}$ has no s-disks, $j([\mathbf{P}])$ is 0 for elements of $\mathcal{B}_{\mathcal{P}}$. Moreover, if $\mathbf{P} \in \mathcal{D}$ then $j([\mathbf{P}])=(\bar{w}-1) \cdot c_{s}^{2}$, where $w$ is in a generating set of $C_{G}(s), s \in \mathbf{s}$. That is, each $\mathbf{P} \in \mathcal{D}$ determines a trivial identity $j([\mathbf{P}])=0$. Thus, $H_{1}(L)$ is defined by trivial identities, and $(\mathcal{Q}, \mathcal{P})$ has the generalized identity property. 
Example 5.9. Consider the pair $\mathcal{P}=\left\langle\mathbf{x}:[u, v]^{2}\right\rangle, \quad \mathcal{Q}=\left\langle\mathbf{x}:[u, v]^{2}, u, v\right\rangle$. We know from Example 4.5 that $(\mathcal{Q}, \mathcal{P})$ has the identity property, so it necessarily has the generalized identity property. However, the spherical picture of Figure 5 is a generator of $\pi_{2} Y$ which is not equivalent to a sum of s-dipoles and pictures over $\mathcal{P}$. It follows that $(\mathcal{Q}, \mathcal{P})$ is not $(\mathrm{CA})$, and the converse to the above theorem is false.

We have the following partial converse to Proposition 5.8.

Proposition 5.10. If $H_{2}(L)=0$ and if $(\mathcal{Q}, \mathcal{P})$ has the generalized identity property then $(\mathcal{Q}, \mathcal{P})$ is $(C A)$.

Proof. We show that any spherical picture $\mathbf{P}$ over $\mathcal{Q}$ is in $J\left(\mathcal{B}_{\mathcal{P}} \cup \mathcal{D}^{*}\right)$. Since $(\mathcal{Q}, \mathcal{P})$ has the generalized identity property, $j([\mathbf{P}])=\sum h_{i}\left(\bar{w}_{i}-1\right) c_{s_{i}}^{2}$ where $h_{i} \in H$, $w_{i} \in C_{G}\left(s_{i}\right)$, and $\bar{w}_{i}$ is the image of $w_{i}$ in $H$. trivial identities in $H_{1}(L)$. We may associate to this sum a natural picture $\mathbf{P}^{\prime}$ over $\mathcal{Q}$ that is a sum of s-dipoles. In particular, the term $h_{i}\left(\bar{w}_{i}-1\right) c_{s_{i}}^{2}$ gives rise to the s-dipole $v_{i} \cdot \mathbf{P}_{w_{i}}$ where $v_{i}$ represents $h_{i}$ in the free group, and $\mathbf{P}_{w_{i}}$ is the s-dipole in $\mathcal{D}^{*}$ associated to $w_{i}$. Let $\mathbf{P}^{\prime}=\sum v_{i} \cdot \mathbf{P}_{i}$. Then $j([\mathbf{P}])=j\left(\left[\mathbf{P}^{\prime}\right]\right)$ and $\left[\mathbf{P}-\left(\mathbf{P}^{\prime}\right)\right] \in$ ker $j=i m i$, where $i: H_{2}\left(X_{L}\right) \rightarrow \pi_{2} Y$ is from the fundamental sequence (1). Now, the Hopf sequence $\pi_{2} X \rightarrow H_{2} X_{L} \rightarrow H_{2} L \rightarrow 0$ ensures that $H_{2} L$ is isomorphic to $H_{2} X_{L} / i m\left(\pi_{2} X\right)$. If $H_{2} L=0$ then every spherical picture over $\mathcal{Q}$ in the image of $H_{2} X_{L}$ actually comes from $\pi_{2} X$. Thus, $\left[\mathbf{P}-\mathbf{P}^{\prime}\right] \in \operatorname{im}\left(\pi_{2} X \rightarrow \pi_{2} Y\right)$, and $\mathbf{P}$ is equivalent (rel $\left.\mathcal{P}\right)$ to a spherical picture whose image is in $J\left(\mathcal{D}^{*}\right)$. It follows that $[\mathbf{P}] \in J\left(\mathcal{B}_{\mathcal{P}} \cup \mathcal{D}\right)$.

6. A test for (CA). Consider $\mathcal{Q}=\langle\mathbf{x}: \mathbf{r}, \mathbf{s}\rangle$. For each $s$ in $\mathbf{s}$, let $\exp _{s}(\mathbf{P})=$ the number of $s^{+}$disks in $\mathbf{P}$ - the number of $s^{-}$disks in $\mathbf{P}$. Then $\mathcal{Q}$ has parity in $\mathbf{s}$ if and only if $\exp _{s}(\mathbf{P})=0$ for each $s \in \mathbf{s}$ and each $\mathbf{P}$ over $\mathcal{Q}$. Recall, $\mathcal{Q}$ has parity in $\mathbf{s}$ if and only if $(\mathcal{Q}, \mathcal{P})$ has the $H$-identity property. This is true if and only if $(\mathcal{Q}, \mathcal{P})$ is Cockcroft.

A subset $\mathbf{c}$ of a (multiplicative) abelian group $C$ is called linearly independent if

$$
\prod_{i=1}^{k} c_{i}^{n_{i}}=1 \text { implies } n_{i}=0 \text { for each } i
$$

where $n_{i} \in \mathbf{Z}$, and $c_{1}, c_{2}, \ldots, c_{k}$ are distinct elements of $\mathbf{c}$.

Lemma 6.1. If $\mathbf{s}$ determines a linearly independent set of elements in $H_{1}(G)$, then $(\mathcal{Q}, \mathcal{P})$ is Cockcroft.

Proof. Take any spherical picture $\mathbf{P}$ over $\mathcal{Q}$. Assume $\mathbf{P}$ has s-disks. Then any spray to the s-disks determines an equation

$$
\prod_{\mathbf{s}-\text { disks in } \mathbf{P}} w_{i} s_{i}^{\epsilon_{i}} w_{i}^{-1} \stackrel{F}{=} w
$$

where $w \in R$, and $w_{i} \in F$ for each $i$. Viewing this equation in $G$, we obtain

$$
\prod_{\mathbf{s}-\text { disks in } \mathbf{P}} w_{i} s_{i}^{\epsilon_{i}} w_{i}^{-1} \stackrel{G}{=} 1
$$


and modulo $[G, G]$ we have

$$
\prod_{\mathbf{s}-\text { disks in } \mathbf{P}} s_{i}^{\epsilon_{i}} \stackrel{H_{1}(G)}{=} 1 .
$$

Grouping common bases,

$$
\prod_{s \in \mathbf{S}} s^{\exp _{s}(\mathbf{P}) \stackrel{H_{1}(G)}{=}} 1
$$

The linear independence condition implies that $\exp _{s}(\mathbf{P})=0$ for each $s \in \mathbf{s}$.

In the case $\mathbf{s}$ consists of a single word, we have the following simple sufficient condition for $(\mathcal{Q}, \mathcal{P})$ to be $(\mathrm{CA})$.

Theorem 6.2. Suppose $\mathcal{P}=\langle\mathbf{x}: \mathbf{r}\rangle$ presents the group $G$ and the word $s \in F(\mathbf{x})$ lives in the center of $G$ and has infinite order in $H_{1}(G)$. If we let $\mathcal{Q}=\langle\mathbf{x}: \mathbf{r}, s\rangle$ then $(\mathcal{Q}, \mathcal{P})$ is $(C A)$, and $\pi_{2} Y$ is generated (over $\left.\mathcal{P}\right)$ by the set $\mathcal{D}$ of basic $\mathbf{s}$-dipoles.

Proof. Since $s$ has infinite order in $H_{1}(G), \mathcal{Q}=\langle\mathbf{x}: \mathbf{r}, s\rangle$ has parity in $\mathbf{s}=\{s\}$ by Lemma 6.1. Thus, the image $j([\mathbf{P}])$ of any spherical picture $\mathbf{P}$ over $\mathcal{Q}$ has the form

$$
\Sigma h_{i}\left(\bar{g}_{i}-1\right) c_{s}^{2}
$$

where $h_{i} \in H, g_{i} \in G$, and $\bar{g}_{i}$ is its image in $H$. Since the image of $s$ is in the center of $G, C_{G}(s)=G$, and $j([\mathbf{P}])$ is mapped by $\psi$ to a consequence of trivial identities. Since this holds for all $\mathbf{P}$ over $\mathcal{Q},(\mathcal{Q}, \mathcal{P})$ has the generalized identity property. Furthermore, the two conditions on $s$ ensure that $L$, the normal closure of $s$ in $G$, is infinite cyclic. Thus, $H_{2}(L)$ is trivial and $(\mathcal{Q}, \mathcal{P})$ is $(\mathrm{CA})$ by Proposition 5.10.

Example 6.3. Suppose $\mathcal{P}=\langle a, b:[a, b]\rangle$ and $\mathcal{Q}=\langle a, b:[a, b], s\rangle$ where $s$ is any non-trivial word that does not set $a \stackrel{H}{=} b$. Since $G$ is abelian, $C_{G}(s)=G$ is generated by $a$ and $b$, and by our choice of $s$ these words determine distinct elements of $H$. Also, any such $s$ has infinite order in $G=H_{1}(G)$ so $(\mathcal{Q}, \mathcal{P})$ is $(\mathrm{CA})$ by Theorem 6.2. Finally, $\mathcal{P}$ is aspherical, so $\pi_{2} Y$ is generated by two distinct basic s-dipoles $\mathbf{P}_{a}$ and $\mathbf{P}_{b}$.

For instance, consider the presentation $\mathcal{Z}=\left\langle a, b: s=a^{2} b^{-3}\right\rangle$, of the $(2,3)$ torus knot group. Let $\mathcal{P}=\langle a, b: r=[a, b]\rangle$, and $\mathcal{Q}=\langle a, b: r, s\rangle$. Then $(\mathcal{Q}, \mathcal{P})$ is $(\mathrm{CA})$ and $\pi_{2} Y$ is generated by two $s$-dipoles $\mathbf{P}_{a}$ and $\mathbf{P}_{b}$, as seen in Figure 11 . The pictures are formed from simples choice for the disk pictures $\mathbf{B}_{a}$ and $\mathbf{B}_{b}$.
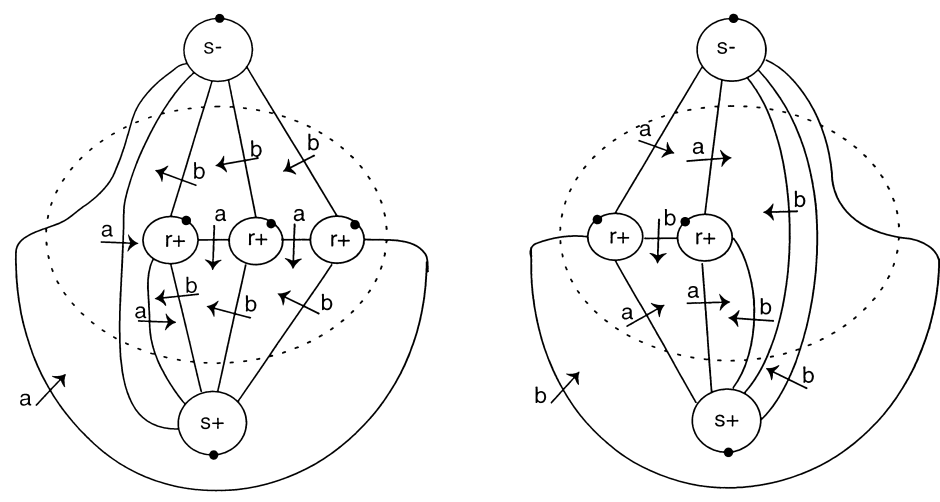

Figure 11 
Example 6.4. Consider the presentation $\mathcal{P}=\left\langle a, b: a^{6}=b^{3}, b^{3}=(a b)^{2}\right\rangle$ of the group $G$, and let $\mathbf{s}=\left\{a^{6}\right\}$. Then $\mathcal{Q}=\left\langle a, b: a^{6}=b^{3}, b^{3}=(a b)^{2}, a^{6}\right\rangle$ presents $H$.

In $G, s$ can be expressed as a product of $a$ 's and as a product of $b$ 's, so $s$ is in the center of $G$. Furthermore, the abelianization of $G$ is the infinite cyclic group generated by $a$, so $a^{6}$ has infinite order in $H_{1}(G)$. Then by Theorem $6.2,(\mathcal{Q}, \mathcal{P})$ is $(\mathrm{CA})$.

To find generators for $\pi_{2} Y$ (over $\mathcal{P}$ ) first note that $C_{G}(s)=G$ since $s$ is in the center of $G$. This centralizer is generated by $a$ and $b$, so $\pi_{2} Y$ is generated (over $\mathcal{P}$ ) by two $s$-dipoles $\mathbf{P}_{a}$ and $\mathbf{P}_{b}$, as shown in Figure 12.
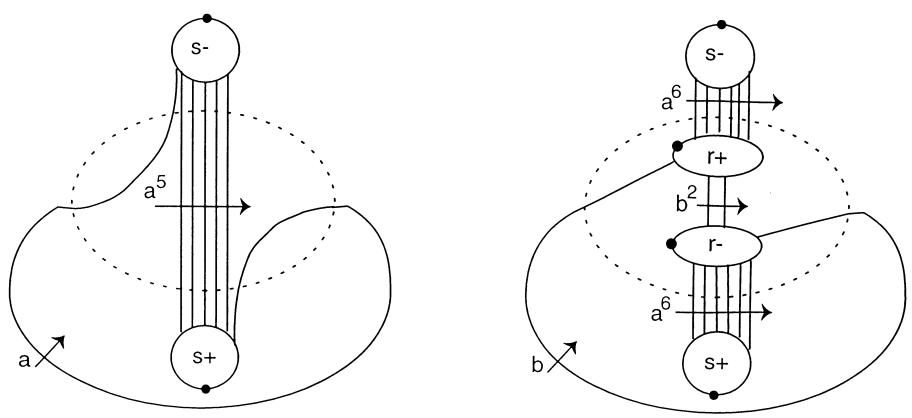

Figure 12

ACKNOWLEDGEMENTs. The author would like to acknowledge the many helpful conversations with Mike Dyer and with Bill Bogley as this paper took shape.

\section{REFERENCES}

1. J. F. Adams, A new proof of a theorem of W.H. Cockcroft, J. London Math. Soc. 30 (1955), 482-488.

2. Y. G. Baik and S. J. Pride, Generators of the second homotopy module of presentations arising from group constructions, preprint (University of Glasgow, 1992).

3. W. A. Bogley, Unions of Cockcroft two-complexes, Proc. Edinburgh Math. Soc. (2) 37 (1994), 317-324.

4. W. A. Bogley, Whitehead's asphericity question, in Two-dimensional homotopy and combinatorial group theory (London Math. Society Lecture Note Series, 197 (1993), edited by C. Hog-Angeloni, W. Metzler and A. J. Sieradski), 309-334.

5. W. A. Bogley and S. J. Pride, Calculating generators of $\pi_{2}$, in Two-dimensional homotopy and combinatorial group theory (London Math. Society Lecture Note Series, 197 (1993), edited by C. Hog-Angeloni, W. Metzler and A. J. Sieradski), 157-188.

6. K. A. Brown, Cohomology of groups (Springer-Verlag, 1982).

7. M. N. Dyer, Crossed modules and $\pi_{2}$ homotopy modules, in Two-dimensional homotopy and combinatorial group theory (London Math. Society Lecture Note Series, 197 (1993), edited by C. Hog-Angeloni, W. Metzler and A. J. Sieradski), 125-156.

8. M. N. Dyer, Adding 2-cells in a delicate way, preprint (University of Oregon, 1993).

9. N. D. Gilbert and J. Howie, Threshold subgroups for Cockcroft 2-complexes, Comm. Algebra 23 (1995), 255-275.

10. M. A. Gutièrrez and J. G. Ratcliffe, On the second homotopy group, Quart. J. Math. Oxford Ser. (2) 32 (1981), 45-55.

11. J. Harlander, Minimal Cockcroft subgroups, Glasgow Math. J. 36 (1994), 87-90.

12. J. Howie, Some remarks on a problem of J.H.C. Whitehead, Topology 22 (1983), 475-485. 
13. S. J. Pride, Identities among relations of group presentations, in Group theory from a geometrical viewpoint, Trieste 1990 (edited by E. Ghys, A. Haefliger, and A. Verjovsky), (World Scientific Publishing, 1991).

14. S. J. Pride, Examples of a presentation which are minimally Cockcroft in several different ways, J. Pure Appl. Algebra 88 (1993), 199-204.

15. J. H. C. Whitehead, On adding relations to homotopy groups, Ann. of Math. 42 (1941), 1197-1239. 